\title{
A Note on Exact Solutions of Coupled (2+1)-dimensional Nonlinear Systems of Schrödinger Equations
}

\author{
Yingqing Song ${ }^{1} \&$ Zaiyun Zhang $^{2}$ \\ ${ }^{1}$ School of Mathematics and Computation Sciences, Hunan City University, Yiyang, Hunan Province, China \\ ${ }^{2}$ School of Mathematics, Hunan Institute of Science and Technology, Yueyang, Hunan Province, China \\ Correspondence: Zaiyun Zhang, School of Mathematics, Hunan Institute of Science and Technology, Yueyang \\ 414006, Hunan Province, China. E-mail: zhangzaiyun1226@126.com
}

Received: July 1, 2012 Accepted: July 26, 2012 Online Published: November 26, 2012

doi:10.5539/jmr.v4n6p121 URL: http://dx.doi.org/10.5539/jmr.v4n6p121

\begin{abstract}
In this paper, we investigate the coupled (2+1)-dimensional nonlinear systems of Schrödinger equations given in Khani, Darvishi, Farmany and Kavitha (2010) and obtain exact traveling solutions by using the modified trigonometric function series method, the modified $\left(\frac{G^{\prime}}{G}\right)$-expansion method and First Integral Method respectively.
\end{abstract}

Keywords: Schrödinger equation, exact solution, modified trigonometric function series method(MTFSM), modified $\left(\frac{G^{\prime}}{G}\right)$-expansion method, First Integral Method (FIM)

PACS numbers: 04.20.Jb, 52.35.Sb, 02.30.Jr

\section{Introduction}

It is well known that traveling wave solutions of Nonlinear Partial Differential Equations (NPDEs) play an important role in the study of nonlinear wave phenomena. The wave phenomena are observed in fluid dynamics, plasma, elastic media, optical fibres, etc. In the recent years, many direct methods have been developed to obtain traveling wave solutions to the nonlinear partial differential equations (NLPDEs), such as the trigonometric function series method (Zhang, 2008; Ma \& Fuchssteiner, 1996), the modified mapping method and the extended mapping method (Zhang, Liu, Miao, \& Chen, 2010), the modified trigonometric function series method (Zhang, Li, Liu, \& Miao, 2011), the dynamical system approach and the bifurcation method (Zhang, Liu, Miao, \& Chen, 2011; Zhang, Gan, \& Yu, 2011), the exp-function method (Yıldırım, \& Pınar, 2010; Khani, Darvishi, Farmany, \& Kavitha, 2010), the multiple exp-function method (Ma, Huang, \& Zhang, 2010), the transformed rational function method (Ma \& Lee, 2009), the symmetry algebra method (consisting of Lie point symmetries) (Ma \& Chen, 2009), the Wronskian technique (Ma \& You, 2005), the modified $\left(\frac{G^{\prime}}{G}\right)$-expansion method (Miao \& Zhang, 2011) and so on.

In this paper, we constuct new exact traveling solutions to the coupled $(2+1)$-dimensional nonlinear systems of Schrödinger equations given in Khani, Darvishi, Farmany and Kavitha (2010).

$$
\begin{gathered}
i \Psi_{t}-\Psi_{x x}+\Psi_{y y}+|\Psi|^{2} \Psi-2 \Psi \Phi=0, \\
\Phi_{x x}-\Phi_{y y}-\left(|\Psi|^{2}\right)_{x x}=0,
\end{gathered}
$$

where $\Psi(x, y, t)$ and $\Phi(x, y, t)$ are complex-valued functions. Nonlinear partial differential equation systems of the type given by (1.1) play an important role in atomic physics, and the functions $\Psi$ and $\Phi$ have different physical meanings in different branches of physics, see Feng (2002); Feng and Wang (2003); Abbasbandy and Shirzadi (2010). More details are presented in Khani, Darvishi, Farmany and Kavitha (2010) and the references therein.

Quite recently, by the trigonometric-function series method (Zhang, 2008) and the exp-function method (Y1ldirım \& Pınar, 2010; Khani, Darvishi, Farmany, \& Kavitha, 2010), Zhang et al. (2011) proposed a new method called the modified trigonometric function series method(MTFSM) to obtain traveling wave solutions to the perturbed nonlinear Schrödinger's equation (NLSE) with Kerr law nonlinearity as follows

$$
i u_{t}+u_{x x}+\alpha|u|^{2} u+i\left[\gamma_{1} u_{x x x}+\gamma_{2}|u|^{2} u_{x}+\gamma_{3}\left(|u|^{2}\right)_{x} u\right]=0 .
$$

In the absence of $\gamma_{1}, \gamma_{2}, \gamma_{3}$ (i.e $\gamma_{1}=\gamma_{2}=\gamma_{3}=0$ ), Equation (1.2) reduces to

$$
i u_{t}+u_{x x}+\alpha|u|^{2} u=0 \text {. }
$$


It is well known that the NLSE (1.3) admits the bright solution solution Feng (2008) or Zhang, Li, Liu and Miao (2011):

$$
u(x, t)=k \sqrt{\frac{2}{\alpha}} \operatorname{sech}(k(x-2 \mu t)) e^{i\left[\mu x-\left(\mu^{2}-k^{2}\right) t\right]},
$$

where $\alpha$ and $k$ are arbitrary real constants, for the self-focusing case $\alpha>0$, and the dark soliton solution Lu, Cui, and Jiang (2007) or Zhang, Li, Liu, and Miao (2011):

$$
u(x, t)=k \sqrt{-\frac{2}{\alpha}} \tanh (k(x-2 \mu t)) e^{i\left[\mu x-\left(\mu^{2}+2 k^{2}\right) t\right]},
$$

where $\alpha$ and $k$ are arbitrary real constants, for the de-focusing case $\alpha<0$. For related problem, we can see Ding and $\mathrm{Li}$ (1996) and Feng (2003) and the references therein.

It is worth mentioning that Zhang, Liu, Miao and Chen (2010); Zhang, Li, Liu and Miao (2011); Zhang, Liu, Miao and Chen (2011); Zhang, Gan and Yu (2011) and Miao and Zhang (2011) considered the NLSE with Kerr law nonlinearity and obtained some new exact traveling wave solutions of Equation (1.2). In Zhang, Li, Liu and Miao (2011), by using the modified trigonometric function series method, Zhang et al. studied some new exact traveling wave solutions. In Zhang, Liu, Miao and Chen (2010), by using the modified mapping method and the extended mapping method, Zhang et al. derived some new exact solutions of Equation (1.2), which are the linear combination of two different Jacobi elliptic functions and investigated the solutions in the limit cases. In Zhang, Liu, Miao and Chen (2011), by using the dynamical system approach, Zhang et al. obtained the traveling wave solutions in terms of bright and dark optical solitons and the cnoidal waves. The authors found that NLSE with Kerr law nonlinearity has only three types of bounded traveling wave solutions, namely, bell-shaped solitary wave solutions, kink-shaped solitary wave solutions and Jacobi elliptic function periodic solutions. Furthermore, we pointed out the region which these periodic wave solutions lie in. We established the relation between the bounded traveling wave solution and the energy level $h$. We noticed that these periodic wave solutions tend to the corresponding solitary wave solutions as $h$ increases or decreases. Finally, for some special selections of the energy level $h$, it is shown that the exact periodic solutions evolute into solitary wave solution. In Miao and Zhang (2011), by using the modified $\left(\frac{G^{\prime}}{G}\right)$-expansion method, Miao and Zhang derived the traveling wave solutions of Equation (1.2), which are expressed by the hyperbolic functions, trigonometric functions and rational functions. In Zhang, Gan and Yu (2011), by using the theory of bifurcations, Zhang et al. investigated the bifurcations and dynamic behavior of traveling wave solutions to the Equation (1.2). Under the given parametric conditions, all possible representations of explicit exact solitary wave solutions and periodic wave solutions are established. In Zhang, Gan, Yu, Zhang and Li (2012), we investigated nonlinear the perturbed nonlinear Schrödinger's equation (NLSE) with Kerr law nonlinearity given in Zhang, Liu, Miao and Chen (2010) and obtain exact traveling solutions by using Infinite Series Method (ISM), Cosine-Function Method (CFM). We show that the solutions by using ISM and CFM are equal. Finally, we get abundant exact traveling wave solutions of NLSE by using Jacobi elliptic function expansion method (JEFEM).

Remark 1.1 There are some related works on integrable properties of general systems of Schrödinger equations and derivative Schrödinger equations (see Ma \& Zhou, 1999a). A combined system of Schrödinger equations and derivative Schrödinger equations was discussed in detail (see Ma \& Zhou, 1999b). On the other hand, the Duffing equation brings chaotic solutions as well and so exposes diversity of exact solutions to nonlinear equations (see Ma, 2003), which is an interesting phenomenon.

\section{Exact Traveling Wave Solutions of (1.1)}

In this section, we investigate the coupled $(2+1)$-dimensional nonlinear systems of Schrödinger equations given in Khani, Darvishi, Farmany and Kavitha (2010) and obtain new exact traveling solutions by using the modified trigonometric function series method, the modified $\left(\frac{G^{\prime}}{G}\right)$-expansion method and First Integral Method respectively.

To facilitate further on our analysis, we assume that Equation (1.1) has traveling wave solutions in the form Khani, Darvishi, Farmany and Kavitha (2010).

$$
\begin{gathered}
\Psi(x, y, t)=u(\xi) \exp (i \eta), \Phi(x, y, t)=v(\xi), \\
\xi=k(x+l y+2(\alpha-\beta l) t), \eta=\alpha x+\beta y+\gamma t,
\end{gathered}
$$

where $k, l, \alpha, \beta$ and $\gamma$ are constants to be determined. More details are presented in Khani, Darvishi, Farmany and Kavitha (2010). 
Benefited from the ideas of Khani, Darvishi, Farmany and Kavitha (2010), it follows from (1.1) and (2.1) that

$$
\begin{gathered}
k^{2}\left(l^{2}-1\right) u^{\prime \prime}+\left(\alpha^{2}-\beta^{2}-\gamma\right) u+u^{3}-2 u v=0, \\
\left(l^{2}+1\right) v^{\prime \prime}-\left(u^{2}\right)^{\prime \prime}=0
\end{gathered}
$$

Integrating (2.3) with respect to $\xi$ and setting the constants of integration equal to zero, we get

$$
v=\frac{u^{2}}{l^{2}+1} \text {. }
$$

Substituting (2.4) into (2.2), we obtain

$$
k^{2}\left(l^{2}-1\right) u^{\prime \prime}+\left(\alpha^{2}-\beta^{2}-\gamma\right) u+\frac{l^{2}-1}{l^{2}+1} u^{3}=0
$$

That is,

$$
A u^{\prime \prime}+B u+C u^{3}=0
$$

where $A=k^{2}\left(l^{2}-1\right), B=\alpha^{2}-\beta^{2}-\gamma, C=\frac{l^{2}-1}{l^{2}+1}$.

In what follows, we will discuss the new exact traveling solutions to Equation (2.5) by using the modified trigonometric function series method(MTFSM), the modified $\left(\frac{G^{\prime}}{G}\right)$-expansion method and First Integral Method(FIM) respectively.

Method 1 - MTFSM (Zhang, Li, Liu, \& Miao, 2011)

Based on the modified trigonometric function series method (see Zhang, Li, Liu, \& Miao, 2011), we find the following cases of solutions of (2.5) (except the solution are constants)

\section{Case 1.1}

$$
u_{1,2}(\xi)=\frac{A_{1}}{B_{0}} \sin \theta= \pm \frac{1}{B_{0}} \sqrt{\frac{2 A}{B}} \frac{1}{\cosh \left(\xi+\eta_{0}\right)}
$$

\section{Case 1.2}

$$
u_{3,4}(\xi)=\frac{A_{0}+A_{1} \sin \theta}{B_{1} \sin \theta+B_{2} \cos \theta}=\frac{A_{0} \pm A_{1} \frac{1}{\cosh \left(\xi+\eta_{0}\right)}}{ \pm \sqrt{\frac{B}{A}} \sqrt{A_{1}^{2}-A_{0}^{2}} \frac{1}{\cosh \left(\xi+\eta_{0}\right)}-A_{0} \tanh \left(\xi+\eta_{0}\right)}\left(\left|A_{1}\right|>\left|A_{0}\right|\right)
$$

\section{Case 1.3}

$$
u_{5,6}(\xi)=\frac{A_{0}}{B_{2} \cos \theta}=\mp \sqrt{\frac{B}{A}} \frac{A_{0}^{2}}{B_{2}} \frac{1}{\tanh \left(\xi+\eta_{0}\right)} ;
$$

\section{Case 1.4}

$$
u_{7,8}(\xi)=\frac{A_{1} \sin \theta+A_{2} \cos \theta}{B_{0}+B_{1} \sin \theta}=\frac{ \pm A_{1} \frac{1}{\cosh \left(\xi+\eta_{0}\right)}-A_{2} \tanh \left(\xi+\eta_{0}\right)}{ \pm \sqrt{\frac{C}{B}} A_{0} \mp \frac{1}{\cosh \left(\xi+\eta_{0}\right)}}
$$

\section{Case 1.5}

$$
u_{9,10}(\xi)=\frac{A_{2} \cos \theta}{B_{0}}= \pm \sqrt{\frac{B}{A}} \tanh \left(\xi+\eta_{0}\right)
$$

\section{Case 1.6}

$$
u_{11,12}(\xi)=\frac{A_{0}+A_{2} \cos \theta}{B_{0}+B_{1} \sin \theta+B_{2} \cos \theta}=\frac{A_{0}-A_{2} \tanh \left(\xi+\eta_{0}\right)}{ \pm \sqrt{\frac{C}{B}} A_{2} \pm \sqrt{\frac{B}{A}} \sqrt{A_{2}^{2}-A_{0}^{2}} \frac{1}{\cosh \left(\xi+\eta_{0}\right)}-\sqrt{\frac{C}{B}} A_{0} \tanh \left(\xi+\eta_{0}\right)}\left(\left|A_{2}\right|>\left|A_{0}\right|\right) .
$$

In what follows, $|\Psi|$ is the norm of $\Psi, \eta_{0}$ is a constant. From (2.6)-(2.11) and (2.1)(2.4), we obtain the following traveling solutions to Equation (1.1)

$$
\left|\Psi_{1,2}(x, t)\right|=\left|\frac{1}{B_{0}} \sqrt{\frac{2 A}{B}} \frac{1}{\cosh \left((k(x+l y+2(\alpha-\beta l) t))+\eta_{0}\right)}\right| ;
$$




$$
\begin{aligned}
& \left|\Phi_{1,2}(x, t)\right|=\left|\frac{u_{1,2}^{2}}{l^{2}+1}\right| . \\
& \left|\Psi_{3,4}(x, t)\right|=\left|\frac{A_{0} \pm A_{1} \frac{1}{\cosh \left(\left(k(x+l y+2(\alpha-\beta) t)+\eta_{0}\right)\right.}}{ \pm \sqrt{\frac{B}{A}} \sqrt{A_{1}^{2}-A_{0}^{2}} \frac{1}{\cosh \left(\left(k(x+l y+2(\alpha-\beta l) t)+\eta_{0}\right)\right.}-A_{0} \tanh \left(k(x+l y+2(\alpha-\beta l) t)+\eta_{0}\right)}\right|\left(\left|A_{1}\right|>\left|A_{0}\right|\right) ; \\
& \left|\Phi_{3,4}(x, t)\right|=\left|\frac{u_{3,4}^{2}}{l^{2}+1}\right| . \\
& \left|\Psi_{5,6}(x, t)\right|=\left|\sqrt{\frac{B}{A}} \frac{A_{0}^{2}}{B_{2}} \frac{1}{\tanh \left(k(x+l y+2(\alpha-\beta l) t)+\eta_{0}\right)}\right| ; \\
& \left|\Phi_{5,6}(x, t)\right|=\left|\frac{u_{5,6}^{2}}{l^{2}+1}\right| . \\
& \left|\Psi_{7,8}(x, t)\right|=\left|\frac{ \pm A_{1} \frac{1}{\cosh \left(k(x+l y+2(\alpha-\beta l) t)+\eta_{0}\right)}-A_{2} \tanh \left(k(x+l y+2(\alpha-\beta l) t)+\eta_{0}\right)}{ \pm \sqrt{\frac{B}{A}} A_{0} \mp \frac{1}{\cosh (k(x+l y+2(\alpha-\beta l) t)+\eta)}}\right| \\
& \left|\Phi_{7,8}(x, t)\right|=\left|\frac{u_{7,8}^{2}}{l^{2}+1}\right| \text {. } \\
& \left|\Psi_{9,10}(x, t)\right|=\left|\sqrt{\frac{B}{A}} \tanh \left(k(x+l y+2(\alpha-\beta l) t)+\eta_{0}\right)\right| ; \\
& \left|\Phi_{9,10}(x, t)\right|=\left|\frac{u_{9,10}^{2}}{l^{2}+1}\right| .
\end{aligned}
$$

$\left|\Psi_{11,12}(x, t)\right|=$

$$
\begin{gathered}
\left|\frac{A_{0}-A_{2} \tanh \left(k(x+l y+2(\alpha-\beta l) t)+\eta_{0}\right)}{ \pm \sqrt{\frac{B}{A}} A_{2} \pm \sqrt{\frac{B}{A}} \sqrt{A_{2}^{2}-A_{0}^{2}} \frac{1}{\cosh \left(k\left(x+l y+2(\alpha-\beta l) t+\eta_{0}\right)\right.}-\sqrt{\frac{B}{A}} A_{0} \tanh \left(k(x+l y+2(\alpha-\beta l) t)+\eta_{0}\right)}\right|\left(\left|A_{2}\right|>\left|A_{0}\right|\right) . \\
\left|\Psi_{9,10}(x, t)\right|=\left|\frac{u_{9,10}^{2}}{l^{2}+1}\right| .
\end{gathered}
$$

Method 2 - The Modified $\left(\frac{G^{\prime}}{G}\right)$-Expansion Method (Miao \& Zhang, 2011)

Based on the modified $\left(\frac{G^{\prime}}{G}\right)$-expansion method (see Miao \& Zhang, 2011), we find the following cases of solutions of (2.5).

Case 2.1 If $\frac{B}{A}>0$ and $\frac{A}{C}<0$, then we obtain

$$
\begin{gathered}
u_{1}(\xi)= \pm \sqrt{-\frac{B}{C}} \frac{A_{1} \sinh \frac{1}{2} \sqrt{\frac{2 B}{A}} \xi+A_{2} \cosh \frac{1}{2} \sqrt{\frac{2 B}{A}} \xi}{A_{1} \cosh \frac{1}{2} \sqrt{\frac{2 B}{A}} \xi+A_{2} \sinh \frac{1}{2} \sqrt{\frac{2 B}{A}} \xi} \\
u_{2}(\xi)= \pm \sqrt{-\frac{B}{2 C}}\left[\frac{-A_{1} \sinh \frac{1}{2} \sqrt{\frac{B}{A}} \xi+A_{2} \cosh \frac{1}{2} \sqrt{\frac{B}{A}} \xi}{A_{1} \cosh \frac{1}{2} \sqrt{\frac{B}{A}} \xi+A_{2} \sinh \frac{1}{2} \sqrt{\frac{B}{A}} \xi}+\left(\frac{-A_{1} \sinh \frac{1}{2} \sqrt{\frac{B}{A}} \xi+A_{2} \cosh \frac{1}{2} \sqrt{\frac{B}{A}} \xi}{A_{1} \cosh \frac{1}{2} \sqrt{\frac{B}{A}} \xi+A_{2} \sinh \frac{1}{2} \sqrt{\frac{B}{A}} \xi}\right)^{-1}\right], \\
u_{3}(\xi)= \pm \sqrt{-\frac{B}{4 C}}\left[\frac{-A_{1} \sinh \frac{1}{2} \sqrt{\frac{B}{2 A}} \xi+A_{2} \cosh \frac{1}{2} \sqrt{\frac{B}{2 A}} \xi}{A_{1} \cosh \frac{1}{2} \sqrt{\frac{B}{2 A}} \xi+A_{2} \sinh \frac{1}{2} \sqrt{\frac{B}{2 A}} \xi}+\left(\frac{-A_{1} \sinh \frac{1}{2} \sqrt{\frac{B}{2 A}} \xi+A_{2} \cosh \frac{1}{2} \sqrt{\frac{B}{2 A}} \xi}{A_{1} \cosh \frac{1}{2} \sqrt{\frac{B}{2 A}} \xi+A_{2} \sinh \frac{1}{2} \sqrt{\frac{B}{2 A}} \xi}\right)^{-1}\right]
\end{gathered}
$$

Case 2.2 If $\frac{B}{A}<0$ and $\frac{A}{C}<0$, then we obtain

$$
u_{4}(\xi)= \pm \sqrt{\frac{B}{C}} \frac{A_{1} \sinh \frac{1}{2} \sqrt{\frac{2 B}{A}} \xi+A_{2} \cosh \frac{1}{2} \sqrt{\frac{2 B}{A}} \xi}{A_{1} \cosh \frac{1}{2} \sqrt{\frac{2 B}{A}} \xi+A_{2} \sinh \frac{1}{2} \sqrt{\frac{2 B}{A}} \xi}
$$




$$
\begin{gathered}
u_{5}(\xi)= \pm \sqrt{\frac{B}{2 C}}\left[\frac{A_{1} \sinh \frac{1}{2} \sqrt{-\frac{B}{A}} \xi+A_{2} \cosh \frac{1}{2} \sqrt{-\frac{B}{A}} \xi}{A_{1} \cosh \frac{1}{2} \sqrt{-\frac{B}{A}} \xi+A_{2} \sinh \frac{1}{2} \sqrt{-\frac{B}{A}} \xi}-\left(\frac{A_{1} \sinh \frac{1}{2} \sqrt{-\frac{B}{A}} \xi+A_{2} \cosh \frac{1}{2} \sqrt{-\frac{B}{A}} \xi}{A_{1} \cosh \frac{1}{2} \sqrt{-\frac{B}{A}} \xi+A_{2} \sinh \frac{1}{2} \sqrt{-\frac{B}{A}} \xi}\right)^{-1}\right], \\
u_{6}(\xi)= \pm \sqrt{\frac{B}{4 C}}\left[\frac{-A_{1} \sinh \frac{1}{2} \sqrt{-\frac{B}{2 A}} \xi+A_{2} \cosh \frac{1}{2} \sqrt{-\frac{B}{2 A}} \xi}{A_{1} \cosh \frac{1}{2} \sqrt{-\frac{B}{2 A}} \xi+A_{2} \sinh \frac{1}{2} \sqrt{-\frac{B}{2 A}} \xi}-\left(\frac{A_{1} \sinh \frac{1}{2} \sqrt{-\frac{B}{2 A}} \xi+A_{2} \cosh \frac{1}{2} \sqrt{-\frac{B}{2 A}} \xi}{A_{1} \cosh \frac{1}{2} \sqrt{-\frac{B}{2 A}} \xi+A_{2} \sinh \frac{1}{2} \sqrt{-\frac{B}{2 A}} \xi}\right)^{-1}\right] ;
\end{gathered}
$$

Case 2.3 If $\frac{B}{A}=0$ and $\frac{A}{C}<0$, then we obtain

$$
u_{7}(\xi)= \pm \sqrt{-\frac{2 A}{C}} \frac{A_{1}}{A_{1} \xi+A_{2}}
$$

where $A_{1}$ and $A_{2}$ are arbitrary parameters.

Substituting the solutions (2.18)-(2.24) into (2.1), respectively, we get three types of explicit traveling wave solutions of Equation (1.1) as follows:

Case 2.1 If $\frac{B}{A}>0$, then we obtain

$$
\begin{gathered}
\left|\Psi_{1}(\xi)\right|=\left|\sqrt{-\frac{B}{C}} \frac{A_{1} \sinh \frac{1}{2} \sqrt{\frac{2 B}{A}} k(x+l y+2(\alpha-\beta l) t)+A_{2} \cosh \frac{1}{2} \sqrt{\frac{2 B}{A}} k(x+l y+2(\alpha-\beta l) t)}{A_{1} \cosh \frac{1}{2} \sqrt{\frac{2 B}{A}} k(x+l y+2(\alpha-\beta l) t)+A_{2} \sinh \frac{1}{2} \sqrt{\frac{2 B}{A}} k(x+l y+2(\alpha-\beta l) t)}\right|, \\
\left|\Phi_{1}(x, t)\right|=\left|\frac{u_{1}^{2}}{l^{2}+1}\right| . \\
\left|\Psi_{2}(x, t)\right|=\mid \sqrt{-\frac{B}{2 C}}\left[\frac{-A_{1} \sinh \frac{1}{2} \sqrt{\frac{B}{A}} k(x+l y+2(\alpha-\beta l) t)+A_{2} \cosh \frac{1}{2} \sqrt{\frac{B}{A}} k(x+l y+2(\alpha-\beta l) t)}{A_{1} \cosh \frac{1}{2} \sqrt{\frac{B}{A}} k(x+l y+2(\alpha-\beta l) t)+A_{2} \sinh \frac{1}{2} \sqrt{\frac{B}{A}} k(x+l y+2(\alpha-\beta l) t)}\right. \\
\left.+\left(\frac{-A_{1} \sinh \frac{1}{2} \sqrt{\frac{B}{A}} k(x+l y+2(\alpha-\beta l) t)+A_{2} \cosh \frac{1}{2} \sqrt{\frac{B}{A}} k(x+l y+2(\alpha-\beta l) t)}{A_{1} \cosh \frac{1}{2} \sqrt{\frac{B}{A}} k(x+l y+2(\alpha-\beta l) t)+A_{2} \sinh \frac{1}{2} \sqrt{\frac{B}{A}} k(x+l y+2(\alpha-\beta l) t)}\right)^{-1}\right] \mid, \\
\left|\Psi_{3}(x, t)\right|=\mid \sqrt{-\frac{B}{4 C}}\left[\frac{-A_{1}(x, t)|=| \frac{u_{2}^{2}}{l^{2}+1} \mid .}{A_{1} \sinh \frac{1}{2} \sqrt{\frac{B}{2 A}} k(x+l y+2(\alpha-\beta l) t)+A_{2} \cosh \frac{1}{2} \sqrt{\frac{B}{2 A}} k(x+l y+2(\alpha-\beta l) t)}\right. \\
\left.+\left(\frac{A_{1} \sinh \frac{1}{2} \sqrt{\frac{B}{2 A}} k(x+l y+2(\alpha-\beta l) t)+A_{2} \cosh \frac{1}{2} \sqrt{\frac{B}{2 A}} k(x+l y+2(\alpha-\beta l) t)}{A_{1} \cosh \frac{1}{2} \sqrt{\frac{B}{2 A}} k(x+l y+2(\alpha-\beta l) t)+A_{2} \sinh \frac{1}{2} \sqrt{\frac{B}{2 A}} k(x+l y+2(\alpha-\beta l) t)}\right)^{-1}\right] \mid, \\
\left|\Phi_{3}(x, t)\right|=\left|\frac{u_{3}^{2}}{l^{2}+1}\right| .
\end{gathered}
$$

Case 2.2 If $\frac{B}{A}<0$, then we obtain

$$
\begin{gathered}
\left|\Psi_{4}(\xi)\right|==\left|\sqrt{\frac{B}{C}} \frac{A_{1} \sinh \frac{1}{2} \sqrt{-\frac{2 B}{A}} k(x+l y+2(\alpha-\beta l) t)+A_{2} \cosh \frac{1}{2} \sqrt{-\frac{2 B}{A}} k(x+l y+2(\alpha-\beta l) t)}{A_{1} \cosh \frac{1}{2} \sqrt{-\frac{2 B}{A}} k(x+l y+2(\alpha-\beta l) t)+A_{2} \sinh \frac{1}{2} \sqrt{-\frac{2 B}{A}} k(x+l y+2(\alpha-\beta l) t)}\right|, \\
\left|\Phi_{4}(x, t)\right|=\left|\frac{u_{4}^{2}}{l^{2}+1}\right| .
\end{gathered}
$$




$$
\begin{gathered}
\left|\Psi_{5}(\xi)\right|=\mid \sqrt{\frac{B}{2 C}}\left[\frac{A_{1} \sinh \frac{1}{2} \sqrt{-\frac{B}{A}} k(x+l y+2(\alpha-\beta l) t)+A_{2} \cosh \frac{1}{2} \sqrt{-\frac{B}{A}} k(x+l y+2(\alpha-\beta l) t)}{A_{1} \cosh \frac{1}{2} \sqrt{-\frac{B}{A}} k(x+l y+2(\alpha-\beta l) t)+A_{2} \sinh \frac{1}{2} \sqrt{-\frac{B}{A}} k(x+l y+2(\alpha-\beta l) t)}\right. \\
\left.-\left(\frac{A_{1} \sinh \frac{1}{2} \sqrt{-\frac{B}{A}} k(x+l y+2(\alpha-\beta l) t)+A_{2} \cosh \frac{1}{2} \sqrt{-\frac{B}{A}} k(x+l y+2(\alpha-\beta l) t)}{A_{1} \cosh \frac{1}{2} \sqrt{-\frac{B}{A}} k(x+l y+2(\alpha-\beta l) t)+A_{2} \sinh \frac{1}{2} \sqrt{-\frac{B}{A}} k(x+l y+2(\alpha-\beta l) t)}\right)^{-1}\right] \mid, \\
\left|\Phi_{5}(x, t)\right|=\left|\frac{u_{5}^{2}}{l^{2}+1}\right| . \\
\left|\Psi_{6}(\xi)\right|==\mid \sqrt{\frac{B}{4 C}}\left[\frac{-A_{1} \sinh \frac{1}{2} \sqrt{-\frac{B}{2 A}} k(x+l y+2(\alpha-\beta l) t)+A_{2} \cosh \frac{1}{2} \sqrt{-\frac{B}{2 A}} k(x+l y+2(\alpha-\beta l) t)}{A_{1} \cosh \frac{1}{2} \sqrt{-\frac{B}{2 A}} k(x+l y+2(\alpha-\beta l) t)+A_{2} \sinh \frac{1}{2} \sqrt{-\frac{B}{2 A}} k(x+l y+2(\alpha-\beta l) t)}\right. \\
\left.-\left(\frac{A_{1} \sinh \frac{1}{2} \sqrt{-\frac{B}{2 A}} k(x+l y+2(\alpha-\beta l) t)+A_{2} \cosh \frac{1}{2} \sqrt{-\frac{B}{2 A}} k(x+l y+2(\alpha-\beta l) t)}{A_{1} \cosh \frac{1}{2} \sqrt{-\frac{B}{2 A}} k(x+l y+2(\alpha-\beta l) t)+A_{2} \sinh \frac{1}{2} \sqrt{-\frac{B}{2 A}} k(x+l y+2(\alpha-\beta l) t)}\right)^{-1}\right], \\
\left|\Phi_{6}(x, t)\right|=\left|\frac{u_{6}^{2}}{l^{2}+1}\right| .
\end{gathered}
$$

Case 2.3 If $\frac{B}{A}=0$, i.e $B=0(A \neq 0)$, then we obtain the rational function solutions in the form

$$
\begin{gathered}
\left|\Psi_{7}(\xi)\right|=\left|\sqrt{-\frac{2 A}{C}} \frac{A_{1}}{A_{1} k(x+l y+2(\alpha-\beta l) t)+A_{2}}\right|, \\
\left|\Phi_{7}(x, t)\right|=\left|\frac{u_{7}^{2}}{l^{2}+1}\right| .
\end{gathered}
$$

Method 3 - First Integral Method (FIM) (Feng, 2002)

First, we show the basic idea of the First Integral Method (FIM). Next, we will discuss the new exact traveling solutions to Equation (2.5) by using the First Integral Method(FIM).

\section{(1) Basic Idea of the First Integral Method (FIM)}

The pioneer work Feng (2002) introduced the first integral method for a reliable treatment of NPDEs. The useful first interest integral method is widely used by many such as in Feng and Wang (2003); Abbasbandy and Shirzadi (2010); Feng (2008); Lu, Cui and Jiang (2007) and the references therein. To facilitate further on our analysis, we initiate our study by briefly reviewing the procedure. We investigate a general nonlinear partial differential equation(PDE) in the form as follows:

$$
P\left(u, u_{x}, u_{t}, u_{x x}, u_{x t}, u_{t t}, \cdots\right)=0,
$$

where $P$ is a polynomial in its arguments and subscripts denote partial derivatives. By means of the transformation $u=U(\xi), \xi=k x+\omega t+\xi_{0}$, we reduce Equation (2.1) to an ordinary differential equation (ODE) of the form

$$
P\left(U, k U^{\prime}, \omega U^{\prime}, k^{2} U^{\prime \prime}, k \omega U^{\prime \prime}, \omega^{2} U^{\prime \prime}, \cdots\right)=0,
$$

where $k, \omega$ and $\xi_{0}$ are arbitrary constants, $U=U(\xi)$ and the primes denote ordinary derivatives with respect to $\xi$. Next, we introduce a new independent variable

$$
x(\xi)=u(\xi), y=\frac{\partial u(\xi)}{\partial \xi},
$$

which leads to the system of nonlinear ordinary differential equations (ODE)

$$
\frac{\partial x(\xi)}{\partial \xi}=y(\xi), \frac{\partial y(\xi)}{\partial \xi}=F_{1}(x(\xi), y(\xi)) .
$$


Based on the qualitative theory of differential equations (Ding \& Li, 1996), if one can find the first integrals to System (2.35) under the same conditions, the analytic solutions to (2.35) can be solved directly. However, in general, it is difficult to realize this even for a single first integral, because for a given plane autonomous system , there is no general theory telling us how to find its first integrals in a systematic way. A key idea of this approach here to find the first integral is to utilize the Division Theorem. We will apply the Division Theorem to obtain one first integral to (2.35) which reduces (2.33) to a first order integrable ODE. For convenience, first let us recall the Division Theorem for two variables in the complex domain $C$ (Feng, 2008).

Division Theorem: Suppose that $P(w, z), Q(w, z)$ are polynomials in $C(w, z)$ and $P(w, z)$ is irreducible in $C(w, z)$. If $Q(w, z)$ vanishes at all zero points of $P(w, z)$, then there exists a polynomial $G(w, z)$ in $C(w, z)$ such that $Q(w, z)=$ $P(w, z) G(w, z)$.

\section{(2) New Exact Traveling Solutions to Equation (2.5) via FIM}

Using (2.34) and (2.5), we get

$$
\left\{\begin{array}{l}
\frac{\partial x(\xi)}{\partial \xi}=y \\
\frac{\partial y(\xi)}{\partial \xi}=-\frac{B}{A} x-\frac{C}{A} x^{3} .
\end{array}\right.
$$

According to the FIM, we suppose the $x(\xi)$ and $y(\xi)$ are the nontrivial solutions of (2.36) and

$$
Q(x, y)=\sum_{i=0}^{N} a_{i}(x) y^{i}=0
$$

is an irreducible polynomial in the complex domain $C(x, y)$, such that

$$
Q(x(\xi), y(\xi))=\sum_{i=0}^{N} a_{i}(x(\xi)) y^{i}(\xi)=0,
$$

where $a_{i}(x)(i=0,1, \cdots, N)$ are polynomials of $x$ and $a_{N}(x) \neq 0$. Equation (2.37) is called the first integral to (2.36). Owing to the Division Theorem, there exists a polynomial $g(x)+h(x) y \mathrm{n}$ the complex domain in the complex domain $C(x, y)$, such that

$$
\frac{\partial Q}{\partial \xi}=\frac{\partial Q}{\partial x} \frac{\partial x}{\partial \xi}+\frac{\partial Q}{\partial y} \frac{\partial y}{\partial \xi}=(g(x)+h(x) y) \sum_{i=0}^{N} a_{i}(x(\xi)) y^{i}(\xi)=0 .
$$

Suppose that $N=1$, by comparing with the coefficients of $y^{i}(i=0,1)$ from both sides of $(2.38)$, we conclude

$$
\begin{gathered}
\frac{\partial a_{1}(x)}{\partial \xi}=h(x) a_{1}(x), \\
\frac{\partial a_{0}(x)}{\partial \xi}=g(x) a_{1}(x)+h(x) a_{0}(x), \\
a_{1}(x)\left[-\frac{B}{A} x-\frac{C}{A} x^{3}\right]=g(x) a_{0}(x) .
\end{gathered}
$$

We obtain that $a_{1}(x)$ is a constant and $h(x)=0$, taking $a_{1}(x)=1$, and balancing the degrees of $g(x), a_{1}(x)$ and $a_{0}(x)$, we conclude that $\operatorname{deg}(g(x))=1$.

Suppose that $g(x)=A_{1} x+A_{0}$, then we find $a_{0}(x)$ as follows:

$$
a_{0}(x)=c_{1}+A_{0} x+\frac{1}{2} A_{1} x^{2},
$$

where $c_{1}$ is arbitrary integration constant. Substituting $g(x), a_{1}(x)$ and $a_{0}(x)$ into (2.41) and setting all the coefficients of $x$ to be zero, then we obtain a system of nonlinear equations and by solving it, we obtain (for simplicity we take the symbol '+')

$$
A_{0}=0, A_{1}=\sqrt{\frac{2 B}{A}}
$$


It follows from (2.37) and (2.43) that

$$
y=-c_{1}-\sqrt{\frac{2 B}{A}} x^{2} .
$$

Using (2.4) (2.37) and (2.44), we obtain the exact solution to Equation (2.5) as follows

$$
u_{3,1}=-\sqrt{c_{1} \sqrt{\frac{A}{2 B}}} \tan \left[c _ { 1 } \sqrt { \frac { 2 B } { A } } \left(k\left(x+l y+2(\alpha-\beta l) t+\xi_{0}\right] \exp (\alpha x+\beta y+\gamma t)\right.\right.
$$

and then the exact solution to Equation (1.1) can be written as

$$
\begin{gathered}
\left|\Psi_{3,1}(x, t)\right|=\mid-\sqrt{c_{1} \sqrt{\frac{A}{2 B}}} \tan \left[c _ { 1 } \sqrt { \frac { 2 B } { A } } \left(k\left(x+l y+2(\alpha-\beta l) t+\xi_{0}\right] \mid,\right.\right. \\
\left|\Phi_{3,1}(x, t)\right|=\left|\frac{u_{3,1}^{2}}{l^{2}+1}\right|,
\end{gathered}
$$

where $\xi_{0}$ are arbitrary constants.

\section{Conclusion and Discussion}

In this article, to find the traveling wave solutions of nonlinear partial differential equations(NPDEs), we introduce the wave variable $\Psi(x, y, t)=u(\xi) \exp (i \eta), \Phi(x, y, t)=v(\xi)$. So, we obtain the following ordinary differential equation (ODE): $A u^{\prime \prime}(\xi)+B u(\xi)+C u^{3}(\xi)=0$. Indeed, the above equation is the well known the Duffing equation. It is well known that the Duffing equation is the equation governing the oscillations of a mass attached to the end of a spring whose tension(or compression). We can see Feng (2003). Then, we obtain new exact traveling solutions by using the modified trigonometric function series method, the modified $\left(\frac{G^{\prime}}{G}\right)$-expansion method and First Integral Method respectively. Finally, it is worth while to mention that the method can also be applied to solve many other NPDEs in mathematical physics which will be investigated in our another work.

\section{Acknowledgement}

The authors would like to present our sincere thanks to the referee for their valuable and helpful comments and suggestions.

\section{References}

Abbasbandy, S., \& Shirzadi, A. (2010). The first integral method for modified Benjamin-Bona-Mahony equation. Commun. Nonlinear Sci. Numer. Simulat., 15, 1759-1764. http://dx.doi. org/10.1016/j.cnsns.2009.08.003

Feng, Z. S. (2002). On explicit exact solutions to the compound Burgers-KdV equation. Phys. Lett. A, 293, 57-66. http://dx.doi.org/10.1016/S0375-9601(01)00825-8

Feng, Z. S. (2003). Duffing's equation and its applications to the Hirota equation. Phys. Lett. A, 317, $115-119$. http://dx.doi.org/10.1016/j.physleta.2003.08.025

Feng, Z. S. (2008). Travelling wave behavior for a generalized fisher equation. Chaos Soliton Fractals, 38, 481488. http://dx.doi.org/10.1016/j.chaos.2006.11.031

Feng, Z. S., \& Wang, X. H. (2003). The first integral method to the twodimensional Burgers-KdV equation. Phys. Lett. A , 308, 173-178. http://dx.doi.org/10.1016/S0375-9601(03)00016-1

Ding, T. R., \& Li, C. Z. (1996). Ordinary differential equations. Peking: Peking University Press.

Khani, F., Darvishi, M. T., Farmany, A., \& Kavitha, L.(2010). New exact wave solutions of coupled (2+1)dimensional nonlinear systems of Schrödinger equations. ANZIAM J., 52, 110-121. http://dx.doi.org/10.1017/S1446181111000563

Lu, J., Cui, G. Y., \& Jiang, X. S. (2007). Some new exact solutions to the Burgers-Fisher equation and generalized Burgers-Fisher equation. Chin. Phys., 16, 2514-2522. http://dx.doi.org/10.1088/1009-1963/16/9/005

Ma, W. X. (2003). Diversity of exact solutions to a restricted Boiti-Leon-Pempinelli dispersive long-wave system. Phys. Lett. A, 319, 325-333. http://dx.doi.org/10.1016/j.physleta.2003.10.030

Ma, W. X., \& Chen, M. (2009). Direct search for exact solutions to the nonlinear Schrödinger equation. Appl. Math. Comput., 215, 2835-2842. http://dx.doi.org/10.1016/j.amc.2009.09.024 
Ma, W. X., \& Fuchssteiner, B. (1996). Explicit and exact solutions to a Kolmogorov-Petrovskii-Piskunovequation. In. J. Nonlinear Mechanics, 31(3), 329-338. http://dx.doi.org/10.1016/0020-7462(95)00064-X

Ma, W. X., Huang, T. W., \& Zhang, Y. (2010). A multiple exp-function method for nonlinear differential equations and its application. Phys. Scr., 82, 065003. http://dx.doi.org/10.1088/0031-8949/82/06/065003

Ma, W. X., \& Lee, J.-H.(2009). A transformed rational function method and exact solutions to the 3+1 dimensional Jimbo-Miwa equation. Choas, Solitons and Fractals, 42, 1356-1363. http://dx.doi.org/10.1016/j.chaos.2009.03.043

Ma, W. X., \& You, Y. (2005). Solving the Korteweg-DE Vries equation by its bilinear form: wronskian solutions. Trans. Amer. Math. Soc., 357, 1753-1778. http://dx.doi.org/10.1090/S0002-9947-04-03726-2

Ma, W. X., \& Zhou, R. G. (1999a). On inverse recursion operator and tri-Hamiltonian formulation for a KaupNewell system of DNLS equations. Journal of Physics A: Mathematical and General, 32, L239-L242. http://dx.doi.org/10.1088/0305-4470/32/20/102

Ma, W. X., \& Zhou, R. G. (1999b). A coupled AKNS-Kaup-Newell soliton hierarchy. Journal of Mathematical Physics, 40, 4419-4428. http://dx.doi.org/10.1063/1.532976

Miao, X. J., \& Zhang, Z. Y. (2011). The modified $\left(\frac{G^{\prime}}{G}\right)$-method and traveling wave solutions of nonlinear the perturbed nonlinear Schrödinger's equation with Kerr law nonlinearity. Commun. Nonlinear Sci. Numer. Simulat., 16(11), 4259-4267. http://dx.doi.org/10.1016/j.cnsns.2011.03.032

Yildırım, A., \& Pinar, Z.(2010). Application of exp-function method for nonlinear reaction-diffusion equations arising in mathematical biology. Comput. Math. Appl., 60(7), 1873-1880. http://dx.doi.org/10.1016/j.camwa.2010.07.020

Zhang, Z. Y. (2008). New exact traveling wave solutions for the nonlinear Klein-Gordon equation. Turk. J. Phys., $32,235-240$.

Zhang, Z. Y., Gan, X. Y., \& Yu, D. M. (2011). Bifurcation behavior of the traveling wave solutions of nonlinear the perturbed nonlinear Schrödinger's equation with Kerr law nonlinearity. Zeitschrift für Naturforschung A, 66, 721-727. http://dx.doi.org/10.5560/ZNA.2011-0041

Zhang, Z. Y., Gan, X. Y., Yu, D. M., Zhang, Y. H., \& Li, X. P. (2012). A Note on Exact Traveling Wave Solutions of the Perturbed Nonlinear Schrödinger's Equation with Kerr Law Nonlinearity. Commun. Theor. Phys., 57, 764-770. http://dx.doi.org/10.1088/0253-6102/57/5/05

Zhang, Z. Y., Liu, Z. H., Miao, X. J., \& Chen, Y. Z. (2010). New exact solutions to the perturbed nonlinear Schrödinger's equation with Kerr law nonlinearity. Appl. Math. Comput., 216, 3064-3072. http://dx.doi.org/10.1016/j.amc.2010.04.026

Zhang, Z. Y., Liu, Z. H., Miao, X. J., \& Chen, Y. Z. (2011). Qualitative analysis and traveling wave solutions for the perturbed nonlinear Schrödinger's equation with Kerr law nonlinearity. Phys. Lett. A, 375, 1275-1280. http://dx.doi.org/10.1016/j.physleta.2010.11.070

Zhang, Z. Y., Li, Y. X., Liu, Z. H., \& Miao, X. J. (2011). New exact solutions to the perturbed nonlinear Schrodinger's equation with Kerr law nonlinearity via modified trigonometric function series method. Commun. Nonlinear Sci. Numer. Simulat., 16(8), 3097-3106. http://dx.doi.org/10.1016/j.cnsns.2010.12.010 